\title{
Crystallization of novel polyglycine hydrolases found in the fungal response against plant defensive chitinases
}

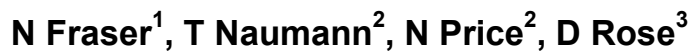 \\ ${ }^{1}$ University of Waterloo, Waterloo, ON, ${ }^{2}$ United States Department of Agriculture (USDA ARS), \\ Peoria, IL, ${ }^{3}$ Dept of Biology, Univ of Waterloo \\ n3fraser@uwaterloo.ca
}

Polyglycine hydrolases (PGH) are a novel class of enzymes that are secreted by fungal families in response to plant-fungal defense; a major component of which are chitinases that target the fungal cell wall without harming the plant. ES-chitinase modifying protein (ES-Cmp) and FS-chitinase modifying protein (FS-Cmp), are two identified PGHs of particular interest that are secreted by Epicoccum sorghi and Fusarium solani respectively. These proteases have a specificity for cleaving N-terminal to glycine-glycine bonds resulting in deactivated plant chitinases. Our work focuses on structure determination of these proteases for inhibitory studies. Despite minimal sequence and motif identity between ES-Cmp and FS-Cmp, both share analogous cleavage activity deviating only in specific glycine recognition. Crystal conditions have been confirmed for FS-Cmp and a native data set was collected to 2.0 angstroms. Current work is focused on crystallizing selenomethionine incorporated proteases to determine phases and solve the structures.

Acta Cryst. (2020). A76, a53 